\title{
THE EFFECT OF MARCHI'S FLUID ON NERVOUS TISSUE THAT HAS UNDERGONE POST-MORTEM CHANGE. ${ }^{1}$
}

By Frederick. E. Batten, M.D.

From the Laboratory of the National Hospital, Queen Square, London.

(Plate XXXV.)

INTRODUCTION.

THE use of osmic acid for the demonstration of early degenerative changes occuring in the nervous system has, in its application to material obtained from experimental sources, given most valuable results. In 1896, however, ${ }^{2}$ Dr. Russell Wells and Dr. W. H. Wilson, in a series of observations, proved that similar appearances could be produced in nervous tissue which had undergone post-mortem change, and that such change might be met with as early as twenty hours after death.

The use of osmic acid in the strength of 1 per cent. gives, however, a result that differs essentially from that obtained by staining nervous tissue by Marchi's fluid, for whereas in the former method the myelin sheath is stained black, in the latter the myelin sheath is of a brown colour, and only when degeneration has taken place does it manifest itself by the presence of black fat granules.

\section{Object of Researcil.}

It was, then, in order to find out to what extent nervous tissue, which had undergone post-mortem change, and had been treated by Marchi's fluid, might give rise to appearances likely to be mistaken for degenerative changes, that the following series of observations were undertaken.

\section{Method of Research.}

In the first case the brain, spinal cord, median, and sciatic nerves

1 Read before the Neurological Society, 28th October 1897.

: Journ. Path. and Bacteriol., Edin. and London, 1896, vol. iii. p. 482. 
were removed from a child æt. 3 , who died of broncho-pneumonia, twenty-four hours after death. The cord was first divided into three parts,-cervical, dorsal, and lumbo-sacral; each of these parts was further divided into six. The sciatic and median nerves were similarly divided into six parts.

Portions of the cord from the cervical, dorsal, and lumbo-sacral region, and a portion of the sciatic and median nerve, were placed in six bottles, between brain tissue, in order to prevent any drying

Müller's fluid was at once added to bottle No. 1; after standing in the laboratory $\left(60^{\circ} \mathrm{F}\right.$.) for twenty-four hours, Müller's fluid was added to bottle No. 2, after forty-eight hours to bottle No 3, after seventy-two hours to bottle No. 4, after ninety-six hours to bottle No. 5 , and after 120 hours to bottle No. 6. The Müller's fluid was changed every day for the first three days, and after that as often as it became cloudy.

After hardening for fourteen days, the tissue was removed from the Müller's fluid and small sections about $\frac{1}{8}$ in. in thickness cut from the cord at the cervical, dorsal, and lumbar region, and placed in Marchi's fluid, the nerves being placed in the same fluid. The tissue was allowed to remain in the solution for five days, was then washed in water for two days, embedded and cut in celloidin in the usual manner, the nerves being cut both longitudinally and transversely.

In the second case the spinal cord and the sciatic nerve were removed from a child, æt. $3 \frac{1}{2}$, sixteen hours after death. The cord was divided in a similar manner to Case 1, into the cervical, dorsal, and lumbo-sacral regions, and each of these subdivided into four parts; the sciatic nerve was also divided into four parts.

Portions from the cord and sciatic nerve were now placed in four bottles between brain tissue, and stood in an incubator, at $40^{\circ} \mathrm{C}$. Müller's fluid was at once added to bottle No. 1, after twelve hours to bottle No. 2, after twenty-four hours to bottle No. 3, and after thirtysix hours to bottle No. 4 . The bottles were then removed from the incubator. The further treatment of the tissue was exactly that described in Case No. 1.

\section{Microscopical Examination.}

In Case No. 1 the sections of the spinal cord taken from the cervical, dorsal, and lumbar region of the spinal cord, which had not been allowed to undergo any further post-mortem change than that which was necessitated by the time after death at which the postmortem could be performed, namely, twenty-four hours, presented a perfectly normal appearance; a few scattered black dots were present in the section, but these gave rise to no appearance that was likely to be mistaken for degeneration. It may be here noted that there is normally in the posterior root, at its entrance into the cord, a con- 
siderable amount of interstitial fat; this also occurs to a lesser degree at the exit of the anterior roots from the cord; an illustration of this condition is shown in Plate XXXV. Fig. 3 ; and, although it has been described as a pathological condition, it is undoubtedly a perfectly normal appearance, in no way due to post-mortem change, or to degeneration of nerve-fibres.

Sections of the cord, twenty-four, forty-eight, seventy-two, ninetysix, and 120 hours after post-mortem present very little change. The sections become progressively more difficult to cut, owing to their friability; the structure of the cord becomes less distinct; and there is some increase in the number of fat globules immediately below the pia mater, though this only occurs ninety-six and 120 hours after post-mortem, and no appearance is produced which is likely to be mistaken for true degeneration.

With regard to the nerves, as with the cord the normal fibres always show a few scattered black dots; even after 120 hours no change is produced which is likely to be mistaken for true degeneration.

In Case 2 the cord and nerves were subjected to a much more severe test; no appearance, however, was produced either in the cord or nerves, that would be likely to be mistaken for degeneration, and that even when, by the Marchi-Pal method, the decomposition change in the nerve could be shown to be most extensive.

The number of scattered black dots on the section of the nerves and spinal cord may appear to be somewhat increased in the tissue in which the greatest amount of change has taken place; and it is to be noted that the myelin sheath sometimes takes the stain more darkly than normal, but does not show the granular fatty appearance of true degeneration.

\section{Conclusions.}

I think that the tests above applied, and the results obtained therefrom, warrant the conclusion that Marchi's fluid will demonstrate degeneration in nervous tissue, even when this cannot be obtained for several hours after death, and may safely be used for that purpose so long as the following points are borne in mind:-

1. That there is normally a considerable amount of interstitial fat at the exit of the anterior roots and the entrance of the posterior roots into the cord (Plate XXXV. Fig. 3).

2. That a few scattered black dots almost always exist, not only in sections of the cord, but also of the nerves, and that such are no evidence of degenerative change.

I would here tender my thanks to the Museum Committee of the National Hospital, Queen Square, for their permission to work in the laboratory of that hospital, and also acknowledge my indebtedness to the pathologist, Dr. Risien Russell, for his kind help and valuable suggestions. 


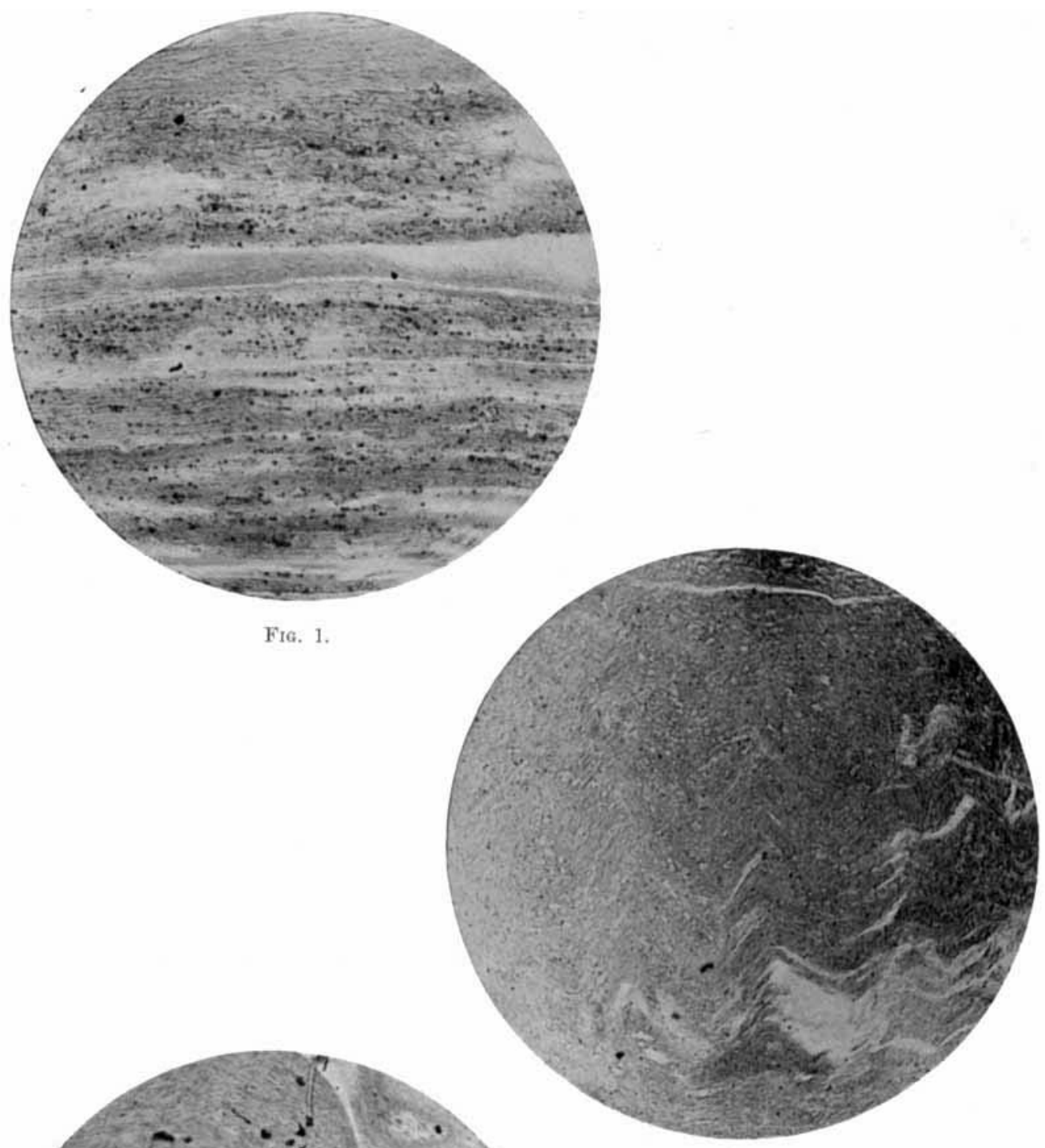

Fie. 2. 


\section{DESCRIPTION OF PLATE XXXV.}

F1G. 1.-Longitudinal section of nerve, stained by Marchi's method, to show typical degeneration.

FIG. 2.-Longitudinal section of nerve (sciatic), in which post-mortem ehange has been allowed to progress for thirty-six hours after removal from the body, at a temperature of $40^{\circ} \mathrm{C}$., to show that although the fibres become broken up, yet it does not give rise to an appearance similar to the typical degeneration, as seen in Fig. 1. The above specimens have been photographed under the same magnifying power.

FIc. 3. - Section through the posterior root at its entrance into the spinal cord, to show fat cells lying among the nerve-fibres, this being a normal condition and not a pathological change. 